\title{
Health status in survivors older than 70 years after hospitalization with COVID-19: observational follow-up study at 3 months
}

\author{
Pamela Carrillo-Garcia ${ }^{1}$ (D) Blanca Garmendia-Prieto ${ }^{1}$. Giovanna Cristofori ${ }^{1}$ - Isabel Lozano Montoya ${ }^{1}$. \\ Javier Jaramillo Hidalgo ${ }^{1}$ - Maribel Quezada Feijoo ${ }^{2}$ • Juan José Baztán Cortés ${ }^{1}$ Javier Gómez-Pavón ${ }^{1}$
}

Received: 4 March 2021 / Accepted: 13 May 2021 / Published online: 31 May 2021

(c) European Geriatric Medicine Society 2021

\section{Key summary points}

Aim To describe associations between functionality, frailty, comorbidity, cognitive and affective status and mortality in a cohort of survivors older patients after hospital admission for SARS-CoV-2 infection.

Findings Severe frailty pre-admission and severe functional dependency at discharge were associated with an increased risk of mortality and readmission at three months. In addition, high comorbidity or the need for readmission was also associated with mortality at 3 months.

Message The knowledge of Health status in older people after hospitalization for COVID-19, means that its early detection can contribute to the selection of patients with greater risk of sequelae in the short term that require more careful follow-up.

\begin{abstract}
Purpose To analyze factors associated with mortality at 3 months and readmissions, functional and cognitive decline, anorexia and affective disorders in patients aged $>70$ years surviving after hospital admission for SARS-CoV-2.

Methods Patients aged $>70$ years, discharged after hospitalization with COVID-19. Outcome variables:mortality, readmissions, functional and cognitive impairment, anorexia and mood disorder.

Results 165 cases at 3 months after hospital discharge, $8.5 \%$ died and $20 \%$ required at least one hospital readmission. The presence of severe dependence at discharge $(\mathrm{BI}<40)$ was associated at 3 months with a higher risk of mortality $(\mathrm{OR} 5.08$; 95\% CI 1.53-16.91) and readmissions (OR 4.53; 95\% CI 1.96-10.49). The post-hospitalization functional deterioration was associated with persistence of deterioration at 3 months (OR 24.57; 95\% CI 9.24-65.39), cognitive deterioration (OR 2.32; 95\% CI 1.03-5.25) and affective (OR 4.40; 95\% CI 1.84-10.55)

Conclusions Loss function in older people after hospitalization by COVID-19 may contribute to identify patients with a higher risk of sequelae in the short term that require closer follow-up.
\end{abstract}

\section{Introduction}

The impact of the SARS-CoV-2 virus in older people has been devastating. According to a report from the National Epidemiological Surveillance Network on the COVID-19 situation in Spain, 37.3\% of the cases reported until 21 May

Pamela Carrillo-Garcia

cpamela312@hotmail.com

1 Department of Geriatric, Hospital Central de la Cruz Roja, San José y Santa Adela, Avenida Reina Victoria 26, 28003 Madrid, Spain

2 Cardiology Department, Hospital Central de la Cruz Roja, San José y Santa Adela, Madrid, Spain
2020 involved individuals aged $>70$ years, representing $87 \%$ of the individuals who died from the virus [1]. There is ample scientific evidence on the mortality and morbidity but there are few data on the medium-term consequences on the health status of older people who have suffered from COVID-19 [2].

Therefore, this brief report aimed to analyse factors associated with mortality at 3 months and readmissions, functional and cognitive decline, anorexia and affective disorders in patients aged $>70$ years surviving after hospital admission for SARS-CoV-2. 


\section{Methods}

Longitudinal observational study of patients $>70$ years old discharged from Hospital Central de la Cruz Roja in Madrid, Spain, from March 20th to May 31st, 2020, with a diagnosis of SARS-CoV-2 infection, defined according to confirmation criteria with Polymerase Chain Reaction-PCR or as suspected cases (on the basis of clinical, imaging and laboratory results). OCTA-COVID Cohort.

\section{Data collection}

At admission, sociodemographic variables (age, sex, and previous institutionalization), comorbidities (Charlson Index, high comorbidity $\geq 3$ ), polypharmacy ( $\geq 5$ drugs; extreme: $\geq 10$ drugs), and the Quick Sepsis related Organ Failure Assessment (qSOFA) score (high risk of mortality in suspected sepsis $\geq 2$ points) were collected from the clinical history. Functional status was assessed using the Barthel Index (BI) (independent: 100, mild: 60-99, moderate: 40-59, severe: 20-39 and maximum dependency: 0-19). Cognitive status was analyzed using the Red Cross Mental Scale (RCMS), which ranges from 0 (no cognitive impairment) to 5 (severe cognitive impairment), considering a score $\geq 2$ as the presence of dementia. Frailty was evaluated using the Clinical Frailty Scale (CFS), categorizing scores 1-4 as non-frail, 5-6 as mildly to moderately frail and 7-9 as severely frail. As an analytical variable, serum albumin levels at admission (normal $>35 \mathrm{~g} / \mathrm{L}$, mild hypoalbuminemia 30-35 g/L and severe hypoalbuminemia $<30 \mathrm{~g} / \mathrm{L}$ ).

At discharge, functional status, length of hospital stay and institutionalization were collected.

At 3 months after discharge, a telephone interview was conducted by a trained geriatrician with the patient or, in the presence of cognitive decline, the reference family member or the nursing home staff. The following outcome variables were collected: mortality (main variable); hospital readmission; functional decline (decrease $\geq 10$ points in the BI compared to the baseline score); cognitive decline (decrease of 1 point on the RCMS compared to the baseline score); depressive semiology (the presentation of one or more of the criteria for the diagnosis of depressive disorder in the Diagnostic and Statistical Manual of Mental Disorders-V (DSM-V)); and anorexia (question A of the Mini Nutritional Assessment (MNA)).

\section{Statistical analysis}

Continuous variables are descriptively summarized using the mean \pm standard deviation (SD), and categorical variables are expressed as percentages. Student's $t$ test was used to compare the quantitative variables, and the $\chi^{2}$ test for categorical variables. The influence of the baseline variables on the outcome variables at 3 months was analyzed by binary logistic regression model, calculating the odds ratio (OR) and the corresponding $95 \%$ confidence interval (CI). The significant baseline variables in the univariate analysis were adjusted for age and sex. The statistical analysis was performed in SPSS 26.0.

\section{Results}

Between March 20 and May 31, 2020, 300 patients $>70$ years were admitted for COVID-19, 176 (58.6\%) were discharged. Of these, 11 were excluded for declining to participate resulting in a sample of 165 . The baseline characteristics are presented in Table 1.

At 3 months, 14 patients died (8.5\%), and $20 \%$ required at least 1 hospital readmission. Of the patients who died, $57 \%$ of the deaths occurred in the first month post-discharge, and the main cause of death in $35.7 \%$ was infectious disease

Table 1 Demographic and clinical characteristics of the sample study of $>70$ years hospitalized with SARS-CoV-2 infection

\begin{tabular}{|c|c|}
\hline & Baseline $(N=165)$ \\
\hline Age (years, SD) & $88.5 \pm 6.73$ \\
\hline Female $(\%)$ & 69.1 \\
\hline Nursing home pre-admission (\%) & 65.6 \\
\hline \multicolumn{2}{|l|}{ CFS* } \\
\hline $1-4$ & 27.3 \\
\hline $5-6$ & 24.8 \\
\hline $7-9$ & 47.9 \\
\hline Charlson Index $\geq 3(\%)$ & 33.9 \\
\hline \multicolumn{2}{|l|}{ Polypharmacy (\%) } \\
\hline$<5$ & 32.1 \\
\hline $5-9$ & 43.6 \\
\hline$\geq 10$ & 24.2 \\
\hline 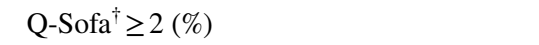 & 13.3 \\
\hline Functional decline pre-admission (\%) & 23.6 \\
\hline $\mathrm{BI}^{\ddagger}$ at discharge (points, SD) & $58.22 \pm 34.32$ \\
\hline Dementia pre-admission $\left(\mathrm{RCMS}^{\S} \geq 2\right)(\%)$ & 43.6 \\
\hline Albumin $(\mathrm{g} / \mathrm{L})$ & $3.34 \pm 0.51$ \\
\hline$<30$ & 21.8 \\
\hline $30-34$ & 36.4 \\
\hline$>35$ & 41.8 \\
\hline Nursing home at discharge (\%) & $69.1 \%$ \\
\hline Length of hospitalization (days) & $15.63 \pm 8.63$ \\
\hline
\end{tabular}


(respiratory and urinary). The most frequent causes of readmission were respiratory $(30.3 \%)$, urinary $(30.3 \%)$ and cardiological (21.2\%) shown in Table 2.

Of the survivors at 3 months, $66 \%$ had the following sequelae: functional decline (27.2\%), cognitive impairment (25.8\%), depressive symptoms (51\%) or anorexia (33.1\%).

In the multivariate analysis adjusted for age and sex, the presence of severe frailty pre-admission and severe functional dependency at discharge were associated with an increased risk of mortality and readmission at 3 months. In addition, high comorbidity or the need for readmission was also associated with mortality at 3 months; the presence of dementia and severe hypoalbuminemia was associated with an increased risk of readmission. Functional decline at discharge, severe hypoalbuminemia and readmission were associated with persistent functional impairment at 3 months (Table 3). Finally, the presence of functional decline at hospital discharge was the only variable that was significantly associated with cognitive impairment at 3 months

Table 2 Mortality and readmissions at 3 months

\begin{tabular}{lc}
\hline Mortality & $8.5 \%(14)$ \\
\hline Heart disease & $7.1 \%$ \\
Respiratory infections & $28.6 \%$ \\
Genitourinary system disease & $7.1 \%$ \\
Unknown & $42.9 \%$ \\
Other & $14.3 \%$ \\
\hline Readmissions & $20 \%(33)$ \\
\hline Heart failure & $21.2 \%$ \\
Respiratory system disease & $30.3 \%$ \\
Genitourinary system disease & $30.3 \%$ \\
Other & $18.2 \%$ \\
\hline
\end{tabular}

$(\mathrm{OR}=2.32 ; 95 \% \mathrm{CI}=1.03-5.25)$ and the presence of depressive symptoms $(\mathrm{OR}=4.40 ; 95 \% \mathrm{CI}=1.84-10.55)$. None of the variables studied were associated with the presence of anorexia at 3 months.

\section{Discussion}

In the present study, we describe the sequelae after hospitalization for COVID-19 in a cohort of patients aged $>70$. One in four patients required readmission or died at 3 months; among the survivors, two out of three patients continued to have functional, cognitive or affective impairment or anorexia.

Mortality at 3 months was $8.5 \%$, lower than that described by Mas Romero et al. in a residential setting (28\%) [3] and lower than the $18.8 \%$ in older patients hospitalized for pneumonia of another cause [4] but only slightly higher than the $6.7 \%$ described at 2 months post-hospitalization in a cohort of COVID-19 patients with a median age of 62 years [5]. Most of the patients in the study had few comorbidities and a baseline situation of mild-moderate dependency that could explain these findings. In addition, the in-hospital mortality of $38 \%$ could have selected the least vulnerable patients with less clinical severity described in a cohort of octogenarians in Spain [6]. According to the literature [7, 8], both frailty and functional dependency were factors associated with mortality and readmission, highlighting the need for early follow-up in this group.

The readmission rate of $20 \%$ was somewhat higher than that described in a sexagenarians cohort, an incidence of $15 \%$ at 2 months was reported [5]. Known pre-pandemic factors such as worse functional, cognitive and nutritional

Table 3 Logistic regression analysis of baseline variables associated to mortality risk, readmission and functional decline at 3 months (adjusted for age and sex)

\begin{tabular}{|c|c|c|c|c|c|}
\hline & Mortality & Readmission & Functional decline & Cognitive impairment & Depressive symptoms \\
\hline Charlson index $\geq 3$ & $3.84(1.17-12.59)$ & $1.81(0.80-4.10)$ & $2.10(0.96-4.58)$ & $1.00(0.44-2.27)$ & $1.13(0.56-2.32)$ \\
\hline CFS* & $7.27(0.86-61.71)$ & $3.07(0.96-9.78)$ & $0.93(0.38-2.23)$ & $1.59(0.63-4.03)$ & $1.12(0.52-2.39)$ \\
\hline $1-4$ & 1 & 1 & 1 & 1 & 1 \\
\hline $5-6$ & $3(0.25-36.06)$ & $1.72(0.44-6.75)$ & $0.87(0.31-2.45)$ & $1.23(0.41-3.68)$ & $1.62(0.66-4)$ \\
\hline $7-9$ & $10.56(1.21-72.51)$ & $4.16(1.25-13.83)$ & $0.96(0.37-2.50)$ & $1.87(0.69-5.06)$ & $0.86(0.38-2)$ \\
\hline Dementia pre-admission & $2.03(0.64-6.42)$ & $2.64(1.16-5.97)$ & $1.50(0.71-3.17)$ & $1.27(0.59-2.70)$ & $0.99(0.51-1.92)$ \\
\hline $\mathrm{BI}^{\dagger}<40$ at discharge & $5.08(1.53-16.91)$ & $4.53(1.96-10.49)$ & $1.58(0.71-3.50)$ & $2.15(0.97-4.77)$ & $0.94(0.46-1.95)$ \\
\hline Hospitalization FD & $1.32(0.39-4.51)$ & $1.53(0.65-3.60)$ & $24.57(9.24-65.39)$ & $2.32(1.03-5.25)$ & $0.23(0.09-0.54)$ \\
\hline Albumin $<30 \mathrm{~g} / \mathrm{L}$ & $1.4(0.40-4.86)$ & $3.35(1.42-7.92)$ & $2.56(1.24-5.86)$ & $0.45(0.16-1.29)$ & $0.49(0.22-1.09)$ \\
\hline Length of hospitalization & $1.02(0.96-1.09)$ & $1.01(0.97-1.06)$ & $1.04(0.99-1.08)$ & $1.00(0.96-1.05)$ & $1.00(0.97-1.04)$ \\
\hline Readmission & $5.88(1.20-12.53)$ & & $3.20(1.33-7.68)$ & & \\
\hline
\end{tabular}

Bold value indicates statistical significance

${ }^{*}$ CFS Clinical Frailty Scale; ${ }^{\dagger} B I$ Barthel Index 
status, were associated with the risk of readmission but not with comorbidity [9, 10].

Functional loss during hospitalization was the only factor associated with functional, cognitive and affective impairment at 3 months. The incidence of post-hospital functional decline at three months after discharge was $27.2 \%$, slightly higher than the $22 \%$ described by Boyd et al. in older people before the pandemic [4]. A quarter of patients worsened cognitively, and half of the patients had some depressive symptoms, an incidence similar to that found by Chopra et al. [5].

This study has limitations derived from being a singlecentre study with a relatively small sample, which requires careful interpretation of the findings. However, the few losses to follow-up (3.5\%) reinforce the internal validity of the study, and the results provide new information on the older people hospitalized for COVID-19.

\section{Conclusions}

COVID-19 results in worsening of health in older people after hospitalization, highlighting the presence of severe functional impairment or the onset of functional decline at discharge, whose early detection could contribute to the selection of patients with a higher risk of sequelae in the short term and the need for closer follow-up.

Acknowledgements Alberto Socorro García, Jorge Artero Ortiz, Luis Tejedor López, Gemma Cuesta Castellón, Lucia Gómez González, Luisa Maria Sánchez Osorio, Sagrario Pérez Delgado, Saleta Maria Goñi Rosón.

\section{Declarations}

Conflicts of interest Authors have no conflicts of interests.

Ethical approval The study was approved by the Clinical Research Ethics Committee of the Hospital Universitario de La Paz, Madrid, registered PI-4131.

\section{References}

1. https://www.isciii.es/QueHacemos/Servicios/VigilanciaSalud PublicaRENAVE/EnfermedadesTransmisibles/Documents/ INFORMES/Informes\%20COVID-19/COVID-19\%20en\%20per sonal $\% 20$ sanitario\%2029\%20de\%20mayo\%20de\%202020.pdf. (Accessed 23 Nov 2020).

2. Richardson S, Carroll C, Close J et al (2020) Research with older people in a world with COVID-19: identification of current and future priorities, challenges and opportunities. Age Ageing 49:901-906. https://doi.org/10.1093/ageing/afaa149

3. Mas Romero M, Avendaño Céspedes A, Tabernero Sahuquillo MT et al (2020) COVID-19 outbreak in long-term care facilities from Spain Many lessons to learn. PLoS ONE 15(10):e0241030. https://doi.org/10.1371/journal.pone.0241030

4. Boyd CM, Landefeld CS, Counsell SR, Palmer RM, Fortinsky RH, Kresevic D, Burant C, Covinsky KE (2008) Recovery of activities of daily living in older adults after hospitalization for acute medical illness. J Am Geriatr Soc 56(12):2171-2179. https://doi.org/10.1111/j.1532-5415.2008.02023.x

5. Chopra V, Flanders SA, O'Malley M, Malani AN, Prescott HC (2020) Sixty-day outcomes among patients hospitalized with COVID-19. Ann Intern Med. https://doi.org/10.7326/M20-5661

6. Ramos-Rincon J-M, Buonaiuto V, Ricci M, Martín-Carmona J, Paredes-Ruíz D, Calderón-Moreno M et al (2020) Clinical characteristics and risk factors for mortality in very old patients hospitalized with COVID-19 in Spain. J Gerontol A Biol Sci Med Sci. https://doi.org/10.1093/gerona/glaa243

7. Geriatric Medicine Research Collaborative (2021) Age and frailty are independently associated with increased COVID-19 mortality and increased care needs in survivors: results of an international multi-centre study. Age Ageing. https://doi.org/10.1093/ageing/ afab026

8. Hewitt, J., Carter, B., Vilches-Moraga, A., Quinn, T. J., Braude, P., Verduri, A., Pearce, L., Stechman, M., Short, R., Price, A., Collins, J. T., Bruce, E., Einarsson, A., Rickard, F., Mitchell, E., Holloway, M., Hesford, J., Barlow-Pay, F., Clini, E., Myint, P. K., ... COPE Study Collaborators (2020) The effect of frailty on survival in patients with COVID-19 (COPE): a multicentre, European, observational cohort study. Lancet Pub Health 5(8):e444e451. https://doi.org/10.1016/S2468-2667(20)30146-8

9. Pedersen MK, Meyer G, Uhrenfeldt L (2017) Risk factors for acute care hospital readmission in older persons in Western countries: a systematic review. JBI Data Syst Rev Implement Rep 15(2):454-485. https://doi.org/10.11124/JBISRIR-2016-003267

10 Ma C, Bao S, Dull P, Wu B, Yu F (2019) Hospital readmission in persons with dementia: a systematic review. Int J Geriatr Psychiatry 34(8):1170-1184. https://doi.org/10.1002/gps.5140

Publisher's Note Springer Nature remains neutral with regard to jurisdictional claims in published maps and institutional affiliations. 